\title{
Come i microbiologi clinici delle marche cercano di togliersi dalla lista delle specie in pericolo di estinzione
}

\author{
Paola Pauri ${ }^{1,2}$,Aldo Manzin ${ }^{2}$, Claudio Maria Maffei ${ }^{3}$ \\ ' GdL EBM AMCLI, Milano; \\ 2 UO Virologia, AOU Ospedali Riuniti, Ancona; \\ ${ }^{3}$ Dipartimento dei Servizi alla Persona e alla Comunità, Regione Marche
}

\section{INTRODUZIONE}

In un'epoca di risorse ridotte come l'attuale, il microbiologo clinico può assumere un nuovo ruolo, ispirato dai principi dell'Evidence Based Medicine (EBM), nel collaborare alla diffusione di un uso più appropriato dei test di laboratorio e nella creazione di idonei profili di assistenza e percorsi diagnostici, nell'ottica del Governo clinico (vedi BOX).

Oltre ad eseguire nel modo migliore e nel minor tempo possibile i test richiesti, il microbiologo viene così a svolgere un ruolo attivo nello stabilire accuratezza e utilità clinica dei test utilizzati, in altri termini l'idoneità a risolvere i quesiti clinici che ne motivano l'uso.

Per percorrere queste nuove vie è spesso necessario un difficile confronto con i clinici, con i colleghi di laboratorio e con altri operatori sanitari. L'intento è favorire la diffusione di interventi e tecnologie atti a fornire al paziente benefici concreti oppure, al contrario, impedirne la diffusione, se più che dalle prove di efficacia, essa è sostenuta dalle consuetudini o da motivazioni culturali o personali.
Nella regione Marche è in atto fin dal 2003 un progetto di razionalizzazione delle attività dei laboratori di microbiologia, che ha coinvolto la delegazione regionale AMCLI e tutti i laboratoristi.

La logica del progetto regionale - specificità riconosciute alla Microbiologia

Il progetto regionale, coordinato dal Dipartimento dei Servizi alla Persona e alla Comunità, ha identificato una serie di direttrici su cui indirizzare gli interventi:

- Rifondazione dei modelli culturali ed organizzativi del laboratorio, con una estensione dell'interesse, finora prevalente per la fase analitica, alla fase pre-analitica e post-analitica.

- Identificazione di grandi margini di miglioramento dell'appropriatezza delle richieste.

- Miglioramento della qualità tecnico-professionale nel settore della Microbiologia, tra l'altro attualmente disperso in troppi piccoli laboratori.

- Riconoscimento del contenuto tecnico-professionale molto specifico della Microbiologia che richiede competenze, requisiti e formazione specialistica.

- Concentrazione di attività a livello zonale e

\section{BOX. Finalità del Governo clinico}

Indirizzare verso pratiche assistenziali di documentata efficacia, favorendone l'uso appropriato e monitorandone l'efficacia (outcome)

Fornire una assistenza integrata e multidisciplinare, in una visione centrata sul paziente e sul suo percorso assistenziale

La realizzazione del governo clinico è condizionata da una corretta gestione della conoscenza, che a sua volta poggia sui pilastri dell' educazione medica continua e dell'aggiornamento professionale. Il fine della traduzione dell'informazione (Knowledge translation) è generare una corretta conoscenza al fine di introdurre l'evidenza scientifica nei singoli interventi sanitari. Il Knowledge brokering, "la mediazione della conoscenza" è qualcosa di più, che va oltre il trasferimento della conoscenza da un docente a un discente.

Il "mediatore delle prove scientifiche", l'evidence broker, opera per individuare e mettere in contatto le persone interessate alla soluzione dello stesso problema e stabilire una rete operativa di aiuto reciproco per giungere a decisioni adeguate alle attuali conoscenze scientifiche. Il broker è quindi l'operatore sanitario che è in grado non solo di trasferire conoscenze, ma anche di organizzare riunioni con i colleghi e con i responsabili della politica sanitaria, aiutandoli a sviluppare progetti basati sull'evidenza scientifica. 
sovra-zonale per economia di scala o complessità di esecuzione o garanzia di elevati livelli di competenza.

Sono stati inoltre fissati principi generali per il miglioramento dell'appropriatezza della richiesta di esami di laboratorio, tenendo conto delle due dimensioni dell'appropriatezza, quella clinica, che comprende la scelta e l'utilizzazione dei risultati del test, e quella organizzativa, che comprende la esecuzione nella sede opportuna, con adeguata organizzazione del lavoro:

Creazione di un nuovo ruolo dei laureati di Laboratorio: Adattamento di Linee Guida Evidence based alla realtà locale.

- Costruzione di rapporti di comunicazione costanti con i medici prescrittori (iniziative di informazione e formazione).

- Implementazione di sistemi centralizzati a livello regionale per l'analisi delle prestazioni effettuate da ciascun laboratorio e monitoraggio dei miglioramenti di appropriatezza ottenuti.

\section{Cosa si sta facendo nelle Marche:}

- Partecipazione dei laboratoristi alla stesura del nuovo Nomenclatore/Tariffario regionale delle prestazioni (in vigore dal $1^{\circ}$ gennaio 2004, con una revisione in vigore dal $1^{\circ}$ gennaio 2005), organizzato in quattro aree principali (Anatomia Patologica, Biologia Molecolare, Chimica Clinica, Microbiologia), in molti settori (per la Microbiologia: microbiologia diretta, sierologia infettivologica, virologia diretta) e in quattro livelli di complessità e considerato come uno degli strumenti regionali per il controllo dell'appropriatezza clinica e organizzativa.

- Attivazione in ambito regionale di un Gruppo di Lavoro (GdL) sulla riorganizzazione delle attività di Microbiologia.

- Attivazione in ambito regionale di sei GdL sull'appropriatezza della richiesta, di cui due nell'ambito della Microbiologia, che riguardano l'uso appropriato degli screening infettivologici e il follow-up delle epatiti croniche.

- Identificazione di punti critici da inserire in un progetto di formazione regionale per il 2004, con la collaborazione della delegazione AMCLI.

\section{Gruppo di Lavoro "Riorganizzazione delle attività di Microbiologia"}

Referente: P. Pauri, Servizio di Virologia, Presidio Umberto I, Azienda Ospedaliero-Universitaria Ospedali Riuniti, Ancona.

In una prima fase il GdL ha svolto un'indagine sull'attività dei laboratori di Microbiologia attualmente operanti nella regione, in totale 21 , rilevando una notevole variabilità sia nelle modalità della richiesta dei test (scheda dedicata agli accertamen- ti microbiologici oppure scheda in comune con gli accertamenti di chimica-clinica), sia nella scelta delle metodiche e delle modalità di esecuzione, sia nelle modalità di refertazione. Si è concordato sulla necessità di rendere più omogenei i comportamenti e gli approcci diagnostici, alla luce delle più recenti raccomandazioni basate sulla EBM e di prestare una maggiore attenzione alla fase preanalitica e post-analitica.

Ne è scaturita la proposta di mettere a punto pannelli diagnostici per diverse patologie, protocolli di lavoro omogenei eflow chart per i test di approfondimento.

Inoltre è emerso il desiderio di approfondire gli aspetti della EBM all'interno del laboratorio di Microbiologia, già affrontati negli anni passati con corsi e seminari, oltre alla necessità di supportare con una formazione prevalentemente pratica il processo di certificazione ISO 9001/2000 a cui i laboratori della regione si stanno avvicinando.

A questo scopo, la delegazione regionale AMCLI, che ha già fatto molto sull'argomento, si è impegnata in un ulteriore sforzo formativo per il 2004, che ha portato ad un progetto regionale in collaborazione con il Dipartimento Servizi alla Persona e alla Comunità avente per oggetto la formazione a supporto del processo di riorganizzazione delle attività di microbiologia nei laboratori della regione Marche. Tale progetto ha portato alla realizzazione nel 2004 di una serie di eventi:

- corso teorico-pratico sul "Sistema per la gestione per la qualità in Microbiologia": 2 repliche a maggio e ottobre 2004 rivolte a tecnici e laureati operanti nei settori della Microbiologia;

- incontro sul "Contributo del laboratorio alle attività di gestione clinica e controllo della tubercolosi" che ha coinvolto anche i clinici e i referenti regionali (giugno 2004);

- corso su "L'approccio alla patologia genitale secondo le linee guida evidence-based" che ha coinvolto il Comitato di Studio AMCLI per le infezioni sessualmente trasmissibili non AIDS e i ginecologi della regione (settembre 2004);

- corso teorico-pratico su "La Medicina basata sulle evidenze all'interno del laboratorio clinico (EBLM)" (corso AMCLI del V incontro regionale di aggiornamento in Medicina di Laboratorio) (novembre 2004);

- sessione comune AMCLI-SIBioC su "L'appropriatezza della fase pre-analitica" (V incontro regionale di aggiornamento in Medicina di Laboratorio) (novembre 2004);

- sessione AMCLI su "L'epatite virale cronica: dal laboratorio alle attuali opzioni terapeutiche" per i problemi legati al monitoraggio dell'infezione delle epatiti B e C (V incontro regionale di aggiornamento in Medicina di Laboratorio) 
(novembre 2004);

Di tutti gli eventi sono disponibili presso il sito www.amclimarche.it le elaborazioni dei questionari di apprendimento e di gradimento.

Il GdL ha concordato inoltre sull'opportunità di ridurre il numero dei laboratori che effettuano la Microbiologia a 16 (13 corrispondenti alle Zone, oltre alle 2 Aziende Ospedaliere e all'INRCA), oltre a favorire la concentrazione di alcune prestazioni a livello sovrazonale o regionale (test di biologia molecolare, diagnostica delle infezioni da micobatteri, parassitologia, test sierologici di conferma, ecc), per la quale sono stati attivati 2 ulteriori GdL.

Sono stati inoltre identificati alcuni punti critici meritevoli di approfondimento, soprattutto per quanto riguarda protocolli di lavoro omogenei, in particolare per la diagnostica di:

1. Sospetta meningite/meningoencefalite: indicazione degli accertamenti emato-chimici e microbiologici da fornire in urgenza; indicazione degli accertamenti da effettuare dopo la conta delle cellule e la colorazione di Gram in urgenza, differenziati a seconda che il liquor sia torbido o limpido; dichiarazione dei tempi entro i quali i diversi referti devono essere disponibili. Allo scopo un GdL del Dipartimento di Malattie Infettive e Microbiologia dell'Azienda Ospedaliera Umberto I ha esaminato le Linee Guida e le raccomandazioni nazionali ed internazionali disponibili, inserendole all'interno di un Profilo di Assistenza per meningiti/meningoencefaliti, con gli obiettivi di: ricercare la pratica migliore EBM, ottimizzare i tempi di intervento, ridurre la variabilità di approccio. Tale profilo, attualmente in uso presso l'Azienda Ospedali Riuniti di Ancona, potrebbe essere esteso a tutta la regione.

2. Patologia genitale maschile e femminile di sospetta origine infettiva: dalla valutazione critica delle motivazioni per la ricerca di Chlamydia trachomatis è risultato che spesso il patogeno è considerato una causa di vaginite e quindi inappropriatamente richiesto in aggiunta ai comuni patogeni vaginali. Al contrario nella regione non è mai stato programmato uno studio di prevalenza su soggetti a medio o alto rischio, né sono stati presi in considerazione $\mathrm{i}$ campioni alternativi meno invasivi o i pool di campioni proposti dalla più recente letteratura (urine, autoprelievo vaginale).

Sono stati raccolti in ambito regionale $i$ dati sul numero degli accertamenti eseguiti (più di 17.000 nel 2003, eseguiti in 18 laboratori), rilevando disomogeneità sia nella entità della richiesta per Zona territoriale che nella percentuale di positività (spesso non confermata con metodo NAAT alternativo), che nella tipologia dei test utilizzati, alcuni dei quali non corrispondenti agli standard attualmente indicati dalla letteratura internazionale.

Un altro problema è dovuto al fatto che sulla stessa impegnativa viene prescritta una batteria di accertamenti che non tiene conto del problema clinico. Diversamente, andrebbero previste flow chart di approccio diagnostico in funzione del problema della paziente, ad esempio: "sospetta vaginite in donna fertile o in adolescente"; "sospetta infertilità"; "sospetta infezione/patologia da Papillomavirus" (sulla base di reperti citologici o colposcopici); ecc...

3. Gestione del catetere urinario e della richiesta di urinocoltura: in ambito regionale il GdL ha rilevato difformità nell'approccio diagnostico e nella refertazione, che richiedono ulteriori approfondimenti per la rilevanza numerica della casistica. L'urinocoltura da sola rappresenta infatti la maggior richiesta per il laboratorio, a fronte di una resa diagnostica bassa (10-20\%). Esiste una vasta letteratura che ha valutato l'uso di test rapidi alternativi, le cui caratteristiche di accuratezza, unite allo studio di fattori di rischio, potrebbero permettere di evitare numerose urinocolture (8), ma che vanno validate in loco, in collaborazione con i clinici. Anche in questo caso dunque esiste un grande margine di miglioramento dell'appropriatezza della richiesta e dell'approccio diagnostico.

4. Sospetta Polmonite di origine comunitaria $(C A P)$ : presso il Dipartimento di Malattie Infettive e Microbiologia dell'Azienda Ospedali Riuniti di Ancona è stato elaborato un "Profilo di Assistenza per le CAP" che si ispira alle più recenti Linee Guida Internazionali e descrive gli interventi diagnostici e terapeutici precisando i livelli di evidenza e la forza delle raccomandazioni. Ad esempio 2 emocolture prima dell'inizio della terapia e la ricerca degli antigeni urinari di Legionella sono considerati di forza A, mentre le ricerche sierologiche sono considerate di forza $\mathrm{B}$ o addirittura $\mathrm{C}$; il test anti HIV è stato invece proposto recentemente con forza B in soggetti a rischio e nei soggetti di età compresa fra 15 e 54 anni di età (IDSA 2003). In generale la letteratura internazionale è concorde nel ritenere che non è necessario o appropriato effettuare uno spettro completo di accertamenti microbiologici su ogni paziente con CAP. Il ricorso agli accertamenti dovrebbe essere guidato dalla severità clinica, dai fattori di rischio di tipo epidemiologico e dalla risposta al trattamento, secondo accordi localmente condivisi fra clinici e microbiologi. Ecco quindi un altro esempio in cui il ruolo del microbiologo è fondamentale nel guidare l'appropriatezza della richiesta, alla luce della utilità diagnostica.

5. Gestione della patologia da Micobatteri: il GdL ha rilevato la difformità di approccio diagnostico 
fra i diversi Laboratori, ha coinvolto il Referente del Comitato di Studio nazionale sui Micobatteri per la stesura di un progetto articolato in livelli ed ha proposto di invitare altri esperti per illustrare realtà più avanzate, ad esempio quella del Veneto. Il progetto tecnico è stato redatto ed è articolato in tre livelli, per ciascuno dei quali vengono descritti i requisiti specifici rispetto alle indicazioni di legge, tenendo conto del peso epidemiologico della tubercolosi nella nostra regione (100-120 casi all'anno) ed è attualmente in esame presso il Dipartimento regionale.

6. Gestione della infezione in gravidanza $(T O R C H)$ : i problemi identificati dal GdL riguardano: il counselling della paziente, da indirizzare ai centri di riferimento ginecologici regionali; i test da utilizzare per gli approfondimenti diagnostici; l'abolizione delle ingiustificate ripetizioni dei test di $1^{\circ}$ livello in caso di positività; 1'assoluta necessità di una omogeneità regionale del referto, problema per il quale sarà organizzato uno specifico incontro che si terrà nel giugno 2005.

Dopo una discussione plenaria a livello regionale, il GdL ha inoltre ribadito quali devono essere i requisiti del personale addetto al Laboratorio di Microbiologia Clinica:

- Personale tecnico e laureato dedicato (nel senso di permanenza presso il settore per un congruo periodo di tempo), che tuttavia partecipa ai turni di reperibilità del laboratorio

- Affiancamento/addestramento del personale di nuova nomina da parte di personale esperto per almeno 1 anno per i laureati e almeno 3 mesi per i tecnici

- Formazione ECM effettuata nel settore specifico (fino ad ottenere nell'anno la maggioranza dei crediti in Microbiologia)

Partecipazione obbligatoria per i laureati ad audit clinici (almeno 3 nell'anno), intendendo con il termine "audit clinici" le riunioni ufficiali e programmate con il personale di uno o più reparti clinici della propria Zona territoriale o Azienda su specifici argomenti inerenti l'appropriatezza della richiesta e l'approccio diagnostico. Si ritiene che per i laureati la eventuale produzione nell'anno di un lavoro scientifico (articolo o poster) su argomenti di microbiologia possa sostituire l'audit.

Inoltre il settore di Microbiologia deve garantire il proprio adeguamento agli standard proposti dall'AMCLI a livello nazionale e regionale (oltre che dai Comitati AMCLI e dai GdL regionali), eventualmente nell'ambito di incontri formativi. Quanto indicato deve essere annualmente documentato al Servizio Assistenza Ospedaliera del Dipartimento.

Sono infine stati esaminati i dati relativi al ciclo 2003-2004 della VEQ interregionale su microbio- logia, parassitologia, micobatteriologia e sierologia infettivologica, in previsione di un incontro regionale effettuato a dicembre 2004. In tale incontro sono stati presentati i dati di concordanza regionale e gli score ottenuti, evidenziando una buona situazione, ma suscettibile di miglioramento soprattutto per quanto riguarda la parassitologia ematica e problemi concentrati solo su pochi campioni. I dati ottenuti sono stati: per la sierologia una concordanza media del $97 \%$ per 19 laboratori partecipanti, per la batteriologia del $85,6 \%$ per 24 laboratori partecipanti, per la micobatteriologia del $95 \%$ per 5 laboratori partecipanti, per la parassitologia del $82 \%$ per 17 laboratori partecipanti.

\section{GdL "Linee guida per il follow-up delle epatiti croniche"}

Coordinatore: A. Manzin, Servizio di Virologia, Azienda Ospedaliero-Universitaria Ospedali Riuniti, Ancona.

L'obiettivo del GdL è stato quello di definire il percorso diagnostico nelle infezioni croniche da $\mathrm{HBV}$ o HCV, in accordo con le più recenti linee guida ed indicazioni delle Conferenze di Consenso nazionali ed internazionali. É stato ritenuto utile stabilire tipo e frequenza più appropriati dei test per il monitoraggio virologico dei pazienti non trattati e di quelli in trattamento con farmaci antivirali, evitando il ricorso a richieste ridondanti e proponendo flow-chart disegnate sul risultato ottenuto dai test in diverse situazioni cliniche.

Il GdL ha infatti riscontrato che i marcatori di infezione e soprattutto la ricerca di HBV-DNA, HCVRNA (qualitativa e quantitativa) e la genotipizzazione di $\mathrm{HCV}$ sono richieste secondo modalità $\mathrm{e}$ tempi non conformi alle indicazioni delle linee guida internazionali.

La continua evoluzione delle conoscenze sull'argomento impongono la revisione e l'aggiornamento interdisciplinare dei protocolli per la diagnosi, il trattamento ed il follow-up dei pazienti. Gli specialisti dei servizi diagnostici possono svolgere un utile ruolo di consulenza per i colleghi clinici.

\section{GdL "Uso appropriato degli screening infetti-} vologici"

Coordinatore: A. Manzin, Servizio di Virologia, Azienda Ospedaliero-Universitaria Ospedali Riuniti, Ancona.

L'obiettivo del GdL è stato quello di formulare raccomandazioni per eseguire la ricerca di marcatori di infezione da HBV, HCV, HIV solo in presenza di precise indicazioni cliniche che ne garantiscano l'appropriatezza, utilizzando l'esperienza del Servizio di Virologia più sotto riportata.

Nella nostra regione gli screening infettivologici per HBV, HCV, HIV sono ancora oggi richiesti in 
previsione di un intervento chirurgico o in occasione del ricovero ordinario in ospedale, indipendentemente dallo stato clinico del paziente e dalla sua storia.

Ciò avviene per abitudine e per errata percezione del rischio infettivo (soprattutto nei reparti chirurgici), nella convinzione che la conoscenza della potenzialità infettante del paziente possa modificare in senso migliorativo il comportamento dell'operatore, non confermata dalla letteratura (5). A questo proposito, un recente studio randomizzato controllato effettuato in USA sull'impatto clinico dei test preoperatori per la cataratta, in cui gli outcome clinici erano rappresentati dai tassi di complicanze post-operatorie evitate e dal numero di interventi cancellati, non ha dimostrato alcun effetto in entrambi i casi (11). Si consideri che la relativa spesa annua per MEDICARE ammonta a 150 milioni di dollari.

I problemi connessi a questo tipo di screening infettivologici dipendono da:

- Scarso valore predittivo positivo dei test, anche di elevata sensibilità e specificità, se nella popolazione generale la prevalenza d'infezione è bassa

- Induzione, in caso di positività, di problemi psicologici ed ansietà del paziente

- Aumento dei costi correlati alla necessità di test di conferma nel caso di positività al test di screening

- Mancanza di impatto reale dei risultati sulla prognosi e trattamento del paziente.

Le raccomandazioni a questo proposito del Piano Nazionale Linee Guida (www.PNLG.it) sono di mettere sempre in atto le "precauzioni universali" per la protezione dell'operatore dal contagio da materiale biologico potenzialmente infetto. In particolare 5 LG internazionali sugli accertamenti pre-operatori, prodotte dal 1996 in poi, sono state esaminate dal CeVEAS (novembre 2001). La sintesi per quanto riguarda gli esami infettivologici, indagati da 3 LG su 5, tutte concordanti fra loro, indica che non è giustificato il ricorso pre-operatorio routinario ad esami per lo screening per patogeni trasmissibili, in assenza di dati anamnestici. Si raccomanda invece di predisporre percorsi definiti per la gestione tempestiva della esposizione occupazionale a materiale biologico potenzialmente infetto e di mettere in atto le dovute misure di profilassi pre- e post- esposizione.
L'esperienza del Servizio di Virologia del Presidio Umberto I, Azienda OspedalieroUniversitaria Ospedali Riuniti di Ancona.

Il problema è stato affrontato a partire dal $\underline{2001}$ con l'esame retrospettivo dei risultati ottenuti nel 2000 per gli screening pre-ricovero e pre-operatorio: 8.349 campioni esaminati di cui 2.984 per HBsAg, 3.219 per HCV, 2.146 per HIV. La stragrande maggioranza dei campioni risultati positivi allo screening si dimostravano invariabilmente negativi ai test di conferma. Si sono pertanto voluti calcolare per ciascun test i parametri derivati (sensibilità, specificità e valori predittivi) ed in particolare i Likelihood Ratio (rapporti di verosimiglianza $=\mathrm{RV}$ ) positivi e negativi. La EBM sottolinea infatti l'importanza degli RV, in quanto permettono di ottenere informazioni più rilevanti per le decisioni cliniche che riguardano pazienti individuali $(2,7)$, che utilizzando il nomogramma di Fagan permettono di passare dalle probabilità pretest alle probabilità post-test. È proprio in questo campo appunto che il Microbiologo è in grado di fornire allo specialista richiedente un valore aggiunto al sospetto clinico, dimostrando l'efficacia clinica del test richiesto. Nel nostro caso gli RV hanno dimostrato probabilità post-test del risultato positivo estremamente basse, in quanto nel caso specifico si tratta di uno screening condotto su popolazioni non a rischio, in cui si parte da una probabilità pre-test molto bassa, che corrisponde alla prevalenza della positività sierologica nella popolazione generale, non a rischio e asintomatica calcolata nella nostra realtà (La salute nelle Marche 2000) (tabella 1).

$\mathrm{Nel} 2 \underline{2002}$ è stata effettuata una ricerca approfondita della bibliografia internazionale sull'appropriatezza dello screening e sono stati distribuiti ad ogni Unità Operativa dell'Azienda Ospedaliera report contenenti il numero degli accertamenti richiesti nel secondo semestre e il relativo costo, oltre alle indicazioni tratte dalle evidenze della letteratura.

All'inizio del $\underline{2003}$ alcune U.O. internistiche hanno deciso autonomamente di sospendere lo screening al momento del ricovero, ma restava lo scoglio dei Chirurghi arroccati nella loro posizione difensiva. Con la collaborazione della Direzione Sanitaria sono state programmate più edizioni di incontri personalizzati per gruppi di U.O., in cui sono state presentate le evidenze, con

Tabella I.

\begin{tabular}{|c|c|c|c|c|c|c|c|}
\hline & Sensibilità \% & Specificità \% & RVP & RVN & prevalenza & $\begin{array}{l}\text { prob.post-test } \\
\text { (risultato pos) }\end{array}$ & $\begin{array}{l}\text { prob. post-test } \\
\text { (risultato neg) }\end{array}$ \\
\hline $\mathrm{HBsAg}$ & 100 & 98,85 & 87 & 0 & 0,01 & $<10 \%$ & circa 0 \\
\hline $\mathrm{HCV}$ & 100 & 99,19 & 123 & 0 & 0,037 & $<10 \%$ & circa 0 \\
\hline HIV & 100 & 99,91 & IIII & 0 & 0,001 & circa $30 \%$ & circa 0 \\
\hline
\end{tabular}


la completa apertura alla discussione.

Il risparmio dovuto a questo progetto è stato di 10.330 euro nei secondi 6 mesi del 2003. Il progetto è stato esteso nel 2004 con le stesse modalità al Presidio Lancisi e si intende estenderlo nel 2005 anche al Presidio Salesi. Attualmente lo screening non viene più richiesto da nessuna delle U.O. dei due Presidi, mentre è stato potenziato lo studio in urgenza dei pazienti fonte di incidente a rischio di trasmissione parenterale per gli operatori sanitari (GdL Azienda Ospedali Riuniti "Indicazioni per la gestione delle esposizioni a materiale biologico").

\section{CONCLUSIONI}

L'esperienza condotta ha lo scopo di dimostrare che una buona strategia può essere quella in cui il ruolo del Laboratorista è di attore protagonista e non di spettatore occasionale e distaccato.

I Microbiologi devono essere in grado di costruire un proprio nuovo ruolo, teso a presidiare e diffondere l'appropriatezza della richiesta, alla luce della EBM, mettendo a disposizione la loro conoscenza delle performance e del significato clinico dei risultati dei test utilizzati, acquisendo capacità tecniche in ambito EBM, partecipando a GdL in ambito aziendale, territoriale e regionale allo scopo di favorire l'implementazione a livello locale di LG internazionali.

In particolare il Laboratorio di Microbiologia non deve più essere considerato l'ESAMIFICIO, volto a produrre più prestazioni senza averne il controllo, bensì deve preoccuparsi di fare la prestazione giusta, nel minor tempo possibile, che serve a risolvere il quesito clinico, confrontandosi continuamente con il Clinico richiedenti.

La letteratura internazionale ha indicato gli interventi differenziati necessari a migliorare 1'appropriatezza della richiesta:

- Adattamento di Linee Guida internazionali EBM alla realtà locale.

- Costruzione di rapporti di comunicazione costanti con i medici prescrittori (iniziative di informazione e formazione).

- Implementazione di sistemi di reporting per i clinici richiedenti e monitoraggio aziendale e regionale.

- Modifica della scheda di richiesta per scoraggiare l'indiscriminata selezione di esami, allo scopo di richiedere indicazioni motivate.

Noi intendiamo andare oltre l'uso appropriato, verso il monitoraggio dell'efficacia. Pertanto il progetto regionale di razionalizzazione della Microbiologia non è stato passivamente subito dai microbiologi delle Marche bensì guidato con l'impegno, le proposte e la formazione. Per il futuro riteniamo che, se vogliamo continuare a mantenerci al di fuori della lista delle specie in via di estinzione, dovremo continuare in ogni modo a percorrere questa strada.

Si ringrazia per la supervisione e i preziosi consigli il Dott. Giocoli, referente del GdL AMCLI EBM.

\section{GRUPPI DI LAVORO MARCHIGIANI}

\section{GdL "Riorganizzazione delle attività di Microbiologia"}

Referente: P. Pauri, Servizio di Virologia, Presidio Umberto I, Azienda Ospedaliero-Universitaria Ospedali Riuniti, Ancona.

Componenti: M. Acetoso, AO S. Salvatore Pesaro; F. Santacroce, Zona 5; G. Orlandi, Zona 9; C. Agostinelli, Zona 7; E. Del prete, Zona 3; P. Frontini, Presidio Salesi, Azienda OspedalieroUniversitaria Ospedali Riuniti; E. Manso, Presidio Umberto I, Azienda Ospedaliero-Universitaria Ospedali Riuniti; A. Migali, Zona 4; A. Politi, Zona 7; S. Rossi, Zona 10, S. Cipriani, Zona 6.

\section{GdL "Linee guida per il follow-up delle epatiti} croniche"

Coordinatore: A. Manzin, Servizio di Virologia, Azienda Ospedaliero-Universitaria Ospedali Riuniti, Ancona. Componenti: M. Acetoso, AO S. Salvatore Pesaro; F. Santacroce, Zona 5; G. Orlandi, Zona 9; T. Temellini, Zona 3.

\section{GdL "Uso appropriato degli screening infetti- vologici"}

Coordinatore: A. Manzin, Servizio di Virologia, Azienda Ospedaliero-Universitaria Ospedali Riuniti, Ancona. Componenti: M. Acetoso, AO S. Salvatore Pesaro; F. Santacroce, Zona 5; L. Lazzari, Zona 1; E. Delprete, Zona 3, G. Ghiandoni Zona 7; M. Di Pietro, Zona 13.

\section{BIBLIOGRAFIA}

1. Barratt A, Irwig L, Galasziou, et al. Recommendations about screening. JAMA, 1999; 281 (21): 2029-34.

2. Barriga P, Smieja R. Likelihood Ratio for Microbiology. J Cl Microb 2001; 39 (6): 2366.

3. Di Bari P, Mancini C, Pallotta MR, et al. La salute nelle Marche 2000. Agenzia Regionale Sanitaria Marche. Gennaio 2000.

4. Downs SM. Technical Report: Urinary Tract Infections in febrile infants and young children. The Urinary tract Subcommittee of the American Academy of Pediatrics Committee on Quality improvement. Pediatrics, 1999; 103 (4): e54.

5. Gerberding JL, Littell C, Tarkington A, et al. Risk of exposure of surgical personnel to patients' blood during surgery at San Francisco General Hospital. N Engl 
J Med 1990; 322: 1788-93.

6. Gestione intraospedaliera del personale HbsAg o anti HCV positivo. Consensus Conference 1999. Giorn It Inf Osp, 2000; 7 (4): 157-69.

7. Giocoli G. Evidence Based Microbiology. J Cl Microb, 2000; 38 (9): 3250-1.

8. Gorelick MH, Shaw KN. Screening tests for Urinary Tract Infections in children: a meta-analysis. Pediatrics, 104 (5): e54, 1999.

9. Riegelman RK, Hirsch RP. Practice Guidelines for the management of community-acquired pneumonia in adults. Infectious Disease Society of America, 2000. How to study a study and test a test: critical reading of medical literature. $\left(4^{\text {th }} \mathrm{Ed}\right)$ Lippincott Williams and Wilkins.

10. Schein OD, Katz J, Bass E, et al. The Value of Routine Preoperative Medical Testing before Cataract Surgery. N Engl J Med 2000; 342: 168-75.

11. The urine dipstick useful to rule out infections. A meta-analysis of the accuracy. BMC Urology, 2004; 4 (1): 4 .

Paola Pauri

A.O. Universitaria Ospedali Riuniti Servizio di Virologia

Via Conca 71 - 60020 Torrette (AN)

Tel.: 071 5964854; Fax 0715964850

E-mail:p.pauri@ao_umbertoprimo_marche.it 